\title{
On the existence and location of cardiac mucosa: an autopsy study in embryos, fetuses, and infants
}

\author{
G De Hertogh, P Van Eyken, N Ectors, J Tack, K Geboes
}

Gut 2003;52:791-796

See end of article for authors' affiliations

\section{Correspondence to:} Dr G De Hertogh,

Universitair Ziekenhuis St

Rafaël, Dienst

Pathologische

Ontleedkunde,

Minderbroedersstraat 12

3000 Leuven, Belgium;

gert.dehertogh@

uz.kuleuven.ac.be

Accepted for publication

29 November 2002
Background: The incidence of gastric cardiac adenocarcinoma has increased in the last decades. Gaining insight into the pathogenesis of this lesion is hampered by the limited knowledge of the origin and histology of cardiac mucosa (CM). Currently, the location, extent, and even the existence of CM are controversial.

Aims: We studied the development of the gastro-oesophageal junction (GOJ) in embryos, fetuses, and infants to clarify if $C M$ is a normal structure at birth and where it is located.

Subjects: Twenty one autopsy cases were evaluated ranging in age from 13 weeks' gestational age (GA) to seven months.

Methods: The distal oesophagus and proximal part of the stomach were embedded entirely. Serial sections were stained with haematoxylin-eosin and alcian blue/periodic acid-Schiff. The following parameters were measured: length of abdominal oesophagus; length of columnar lined oesophagus; length of $C M$; and distance from CM to angle of His.

Results: CM was present in all evaluated sections. Its mean length varied throughout gestation. A maximum value was reached at a GA of 16 weeks $(1.2 \mathrm{~mm})$. After term delivery it was very short (0.3-0.6 mm). CM was proximal to, or straddled, the angle of His in all cases. During gestation, the mucin staining pattern of the $C M$ was to a high degree similar to that of the developing pyloric mucosa. Conclusions: $\mathrm{CM}$ develops during pregnancy and is present at birth as a normal structure. If the angle of His is taken as a landmark for the GOJ, CM is located in the distal oesophagus.
$\mathrm{T}$ incidence of adenocarcinoma of the gastrooesophageal junction (GOJ) and of the gastric cardia has increased dramatically in Western and Northern Europe, North America, and Australasia in the past 40 years. ${ }^{12}$ Consequently, the cardia has become the focus of intense investigation. However, the pathogenesis of gastric cardiac adenocarcinoma is still unclear and identification of precursor lesions is difficult because of the limited knowledge of the origin of the cardiac mucosa (CM). Currently, there is no consensus on the location or extent of the cardia and consequently on the types of mucosa that can be found in it.

Anatomists have applied the term "cardia" to that part of the stomach which lies around the orifice of the tubular oesophagus. There is no anatomical landmark for the distal margin of the so-defined cardia. Its proximal margin is the GOJ which, according to anatomists, is localised at the level of the angle of His. This is the point where the tubular oesophagus joins the saccular stomach. ${ }^{3}$ The most reliable endoscopic landmark for the GOJ is the proximal margin of the longitudinal gastric mucosal folds. When a hiatal hernia is present, the endoscopist can identify the distal end of the tubular oesophagus with the proximal margin of the gastric folds, as the lumen of the bowel widens abruptly at that level. Thus the proximal margin of the gastric folds is located approximately at the level of the angle of His. ${ }^{4}$ Histologists identify the GOJ using the squamocolumnar junction (SCJ). This is the abrupt transition of the squamous oesophageal mucosa to the columnar gastric mucosa. It has been stated that in most normal individuals, the SCJ is just proximal to the level of the angle of His and to the cephalad margins of the gastric folds. ${ }^{4}$ However, as much as $2 \mathrm{~cm}$ of the distal tubular oesophagus may be lined by columnar gastric mucosa in a normal subject. ${ }^{5}$ Histological descriptions of CM make mention of shallow, occasionally cystic, loosely packed coiled glands which are lined exclusively by a mucus secreting epithelium. Between the CM and the fundic gland mucosa, a transition zone is usually present. This junctional mucosa has a mixed histological appearance containing parietal cells either as solitary cells or as small cell groups in the glands. ${ }^{6}$

The precise extent of the CM is not known. According to Hayward, CM lines the distal $2 \mathrm{~cm}$ of the oesophagus and the proximal stomach. ${ }^{7}$ Other authors state that the glandular mucosa of the distal oesophagus is typical CM with variable numbers of specialised gastric cells, thus implying that the segment of exclusively mucus secreting glands can be much shorter than $2 \mathrm{~cm} .{ }^{5}$ In some studies, CM has been defined as containing both pure mucous and mixed glands with parietal cells. ${ }^{89}$ Using this definition, the segment of CM would be expected to be longer than $2 \mathrm{~cm}$.

Recently, the existence of CM as a normal structure has been questioned by Chandrasoma and colleagues. ${ }^{10-13}$ They suggest that CM is abnormal and results from metaplasia of the oesophageal squamous epithelium as a consequence of reflux damage. In contrast, Kilgore and colleagues, ${ }^{14}$ Zhou and colleagues, $^{8}$ and Glickman and colleagues ${ }^{9}$ support the concept of the gastric cardia as a normal structure which is present from birth.

Thus the nature, location, extent, and even the existence of CM are controversial. Therefore, we performed this study to clarify whether CM is present at birth as a normal structure and, if so, to describe its location in relation to adjacent structures.

\section{SUBJECTS AND METHODS}

For this study we used tissue samples obtained from 31 embryonic, fetal, perinatal, and infant autopsies performed between

Abbreviations: GOJ, gastro-oesophageal junction; $C M$, cardiac mucosa; SCJ, squamocolumnar junction; GA, gestational age; $\mathrm{H} \& \mathrm{E}$, haematoxylin-eosin; AB/PAS, alcian blue/periodic acid-Schiff; POM, primitive oesophageal mucosa; PSM, primitive stomach mucosa. 
August 1999 and August 2001 in the Department of Pathology, Leuven University Hospitals. Subjects ranged in age from 13 weeks' gestational age (GA) to seven months after term delivery. Nineteen cases were induced abortions because of the haemolysis-elevated liver enzymes-low platelets syndrome (one case) or for fetal abnormalities detected by chorion villus biopsy (10 cases) or ultrasonography (eight cases). Eight cases were spontaneous abortions due to acute chorionitis (four cases), intrauterine death with unknown cause (three cases), or twintwin transfusion syndrome (one case).

A prematurely born child died at a corrected GA of 34 weeks 3/7 because of necrotising enterocolitis. Another died at a corrected GA of 37 weeks 4/7 due to congenital herpes virus infection. A third child with a complex cardiopathy died at a corrected GA of 41 weeks. An infant aged seven months died from hypovolaemic shock due to severe gastroenteritis. Autopsies were performed between six and 24 hours after death. At autopsy, a careful in situ examination of the GOJ region was carried out (see fig 1A). A hiatal hernia was absent in all cases. The distal oesophagus and stomach were excised in one piece, together with a ring of diaphragmatic muscle (see fig 1B).

\section{Histological assessment}

Specimens were fixed overnight in 10\% formalin. In 24 cases (age range 13 to 23 weeks' GA), the whole specimen was sectioned longitudinally after routine processing. In six cases (age range 24-41 weeks' GA), the specimen was so large that it had to be embedded top down, after which it was sectioned transversally. In one case (an infant aged seven months), the specimen was divided in multiple longitudinal sections which were embedded separately. The paraffin blocks were sectioned completely in all cases. Sections $(5 \mu \mathrm{m})$ at regular intervals were stained by haematoxylin-eosin (HEE) and evaluated microscopically to select sections showing the mucosae of the distal oesophagus and proximal stomach. The number of sections selected varied between 10 (small specimens) and 40 (large specimens). Additional stored sections from all specimens were stained with alcian blue/ periodic acid-Schiff at $\mathrm{pH} 2.5$ and 0.5 (AB/PAS 2.5 and $\mathrm{AB} / \mathrm{PAS} 0.5$ ) to categorise mucins according to a previously described protocol ${ }^{15}$ (table 1$)$. The slides were assessed separately by three pathologists (GDH, PVE, and KG) who were blinded to each other's findings. The mucosae in the GOJ region were categorised as either primitive oesophageal, squamous, primitive stomach, or cardiac type. The primitive oesophageal mucosa (POM) consisted of a stratified columnar epithelium covered by ciliated cells. Primitive stomach mucosa (PSM) was defined as a layer of columnar epithelial cells with no glandular structures present. We accepted as CM a lining composed of foveolar and surface epithelium overlying glandular structures containing no parietal cells. We chose to exclude mixed glands because it is difficult to distinguish between chief cells and mucous cells throughout gestation. ${ }^{16}$ If we had included mixed glands in the cardiac mucosa, it would have been difficult to distinguish between genuine cardiac and fundic mucosa. Thus we chose to define the distal end of the cardiac mucosa by the first parietal cell we encountered.

In all cases, the following parameters were measured: length of the abdominal segment of the oesophagus (defined as the distance from the lower rim of the diaphragm to the angle of His); length of columnar lined oesophagus (defined as the distance from the SCJ to the angle of His); length of $\mathrm{CM}$; distance from the CM to the angle of His; and length of PSM, if present.

Distances were measured along the mucosal surface using a calibrated graticule in the eyepiece of a Leitz DMRB microscope (objectives: Fluotar, eyepieces: L PLAN 10×/25).

In all cases, distances were measured at six points along the oesophageal perimeter. In four cases, 18 measurements per
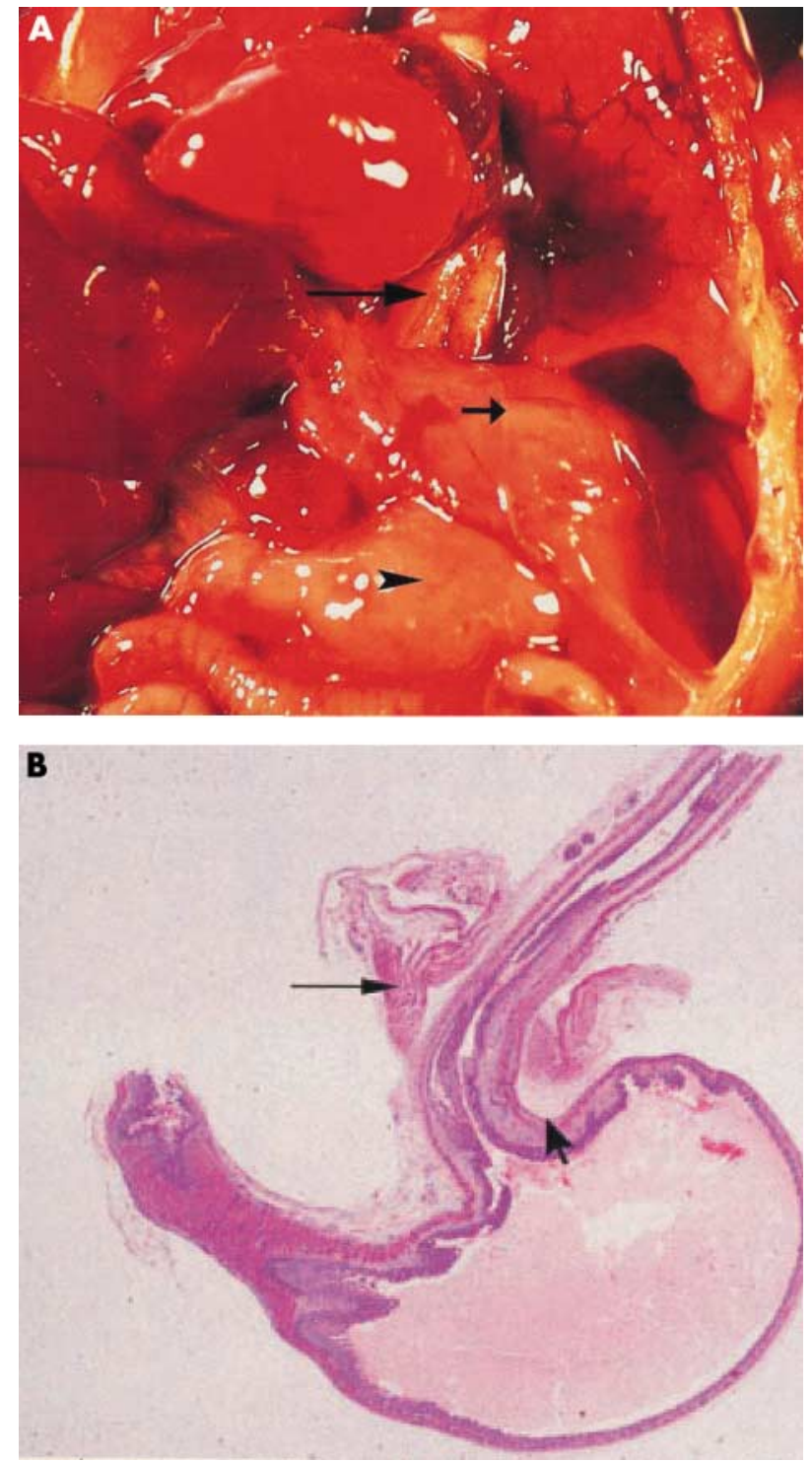

Figure 1 (A) In situ examination of the gastro-oesophageal junction region at autopsy. The long arrow indicates the oesophagus, the short arrow the diaphragm, and the arrowhead the stomach. No hiatal hernia present. (B) Example of a resection specimen (haematoxylin- eosin stained longitudinal section), showing the diaphragm (long arrow) and the angle of His (short arrow).

case were performed to permit the drawing of charts showing the location of the different mucosal types relative to one another and to the diaphragm and the angle of His. Five measuring points were along the greater curvature (GC 1-5), two were located posteriorly ( $\mathrm{P} 1-2$ ), four were along the lesser curvature (LC l-4), three were located anteriorly (A l-3), and four were located between these regions (GC-P between the greater curvature and the posterior stomach wall; P-LC between the posterior stomach wall and the lesser curvature; LC-A between the lesser curvature and the anterior stomach wall; and A-GC between the anterior stomach wall and the greater curvature)

\section{Statistical analysis}

In every case, means were calculated from the six or 18 measurements for each parameter. The means of individual cases were used to calculate mean values for each parameter in groups of cases with the same age. 
Table 1 Mucin staining patterns

\begin{tabular}{lll}
\hline Mucin type & AB/PAS 2.5 & AB/PAS 0.5 \\
\hline Neutral & Magenta & Magenta \\
Sialylated & Aqua & Magenta \\
Neutral and sialylated & Light purple/purple-blue & Magenta \\
Sulphated & Aqua & Aqua \\
Neutral and sulphated & Light purple/purple-blue & Light purple/purple-blue \\
Sialylated and sulphated & Aqua & Light purple/purple-blue \\
\hline AB/PAS, alcian blue/periodic acid-Schiff. & \\
\hline
\end{tabular}

Table 2 Length of the abdominal oesophagus (AO), length of the columnar lined oesophagus (CLO), length of the cardiac mucosa (CM), distance from the CM to the angle of His (CMH), and length of the primitive stomach mucosa (PSM), as a function of age

\begin{tabular}{lllllll}
\hline Age & No of cases & AO & CLO & CM & CMH & PSM \\
\hline 13 w GA & 2 & 1.2 & 0.8 & 0.2 & 0.3 & 0.3 \\
14 w GA & 1 & 0.7 & 0.9 & 0.5 & 0.1 & 0.3 \\
15 w GA & 2 & 1.6 & 1.7 & 1.0 & 0.7 & - \\
16 w GA & 4 & 1.6 & 1.9 & 1.2 & 0.7 & - \\
17 w GA & 1 & 2.5 & 2.7 & 0.9 & 1.8 & - \\
18 w GA & 1 & 1.7 & 1.0 & 1.0 & 0.0 & - \\
19 w GA & 1 & 2.2 & 1.8 & 0.8 & 1.0 & - \\
21 w GA & 3 & 2.4 & 2.5 & 0.8 & 1.7 & - \\
22 w GA & 1 & 3.3 & 2.8 & 0.4 & 2.4 & - \\
23 w GA & 1 & 0.3 & 0.3 & 0.4 & 0.0 & - \\
24 w GA & 2 & 1.8 & 1.4 & 0.4 & 1.0 & - \\
41 w GA & 1 & 0.6 & 0.3 & 0.3 & 1.3 & \\
7 m & 1 & 0.9 & 0.6 & 0.3 & \\
\hline \multicolumn{7}{c}{ Mean values for each age group (mm). } \\
GA, gestational age; w, weeks; months.
\end{tabular}

\section{RESULTS}

Ten cases were excluded because of autolysis. The remaining 21 cases ranged in age from 13 weeks' GA to seven months after term delivery. Table 2 shows the ages of the cases, length of the abdominal oesophagus, length of the columnar lined oesophagus, length of the $\mathrm{CM}$, distance from the $\mathrm{CM}$ to the angle of His, and length of the PSM (mean values for all ages).

Cardiac mucosa was present at a GA of 13 weeks. The mucosal surface and pits were lined by a single layer of tall columnar epithelial cells with elongated basally situated nuclei. The apical cytoplasm was filled with mucus, which was lightly eosinophilic in HEE stained sections. Histochemically, both neutral and sialylated mucins were present. At this early stage, the glands were merely shallow sacs at the base of the pits. A neck part could not be discerned. The epithelial cells lining the glands were cuboidal to triangular and contained a centrally located round nucleus. In H\&E stained sections, the apical cytoplasm of some gland cells contained a small amount of eosinophilic substance. Histochemically, this substance proved to be a mixture of neutral and sialylated mucins. The gland cells could easily be distinguished from parietal cells, which stood out as bright red in H\&E stained sections and stained negative in histochemical stains.

CM was interposed between PSM proximally and mucosa containing parietal cells distally. The PSM consisted of a tall columnar epithelium. The most distal part of this epithelium showed small depressions, in which the nuclei were placed radially at the base of the depression. These structures correspond most likely to early gland pits. Some pit cells in the PSM contained a mixture of neutral and sialylated mucins. H\&E and mucin stains of the CM in an embryo of GA 13 weeks are shown in fig $2 \mathrm{~A}$ and $\mathrm{B}$, respectively. The location of the different mucosal types in this embryo is shown in fig 3.

PSM was present at 13 and 14 weeks only. In fetuses aged 15 weeks, CM linked up with POM without interposition of another mucosal type. At this age, gland cells contained either no mucins or a small amount in comparison with surface and foveolar epithelial cells. The mucins present were neutral, sialylated, or sulphated in various combinations. In contrast, most surface and foveolar cells contained neutral mucins only. This staining pattern was identical to that observed in the developing pyloric mucosa. The most distal part of the oesophagus was covered by mucosa containing parietal cells. In this segment, the surface and foveolar cells had a mucin staining pattern identical to similar cells along the greater curvature (predominantly sialylated mucins).

From 15 to 17 weeks, glands in the CM lengthened considerably. At 17 weeks, most gland cells and the majority of the surface and foveolar epithelial cells contained exclusively neutral mucins. However, in the most proximal glands, gland cells still contained mainly a mixture of neutral and sialylated mucins. From GA 21 weeks onwards, the neck part of the glands could be identified as a constriction between the pit cells and the glandular cells. Several glands opening into one pit were observed. At 23 weeks, the POM was replaced by squamous mucosa with only occasional ciliated cells at the mucosal surface. HEE and mucin stains of the CM in an end second trimester fetus of GA 24 weeks are shown in fig 4A and $\mathrm{B}$, respectively. The location of the different mucosal types in this fetus is shown in fig 5 .

During the last three months of pregnancy, lengthening of all glands and further differentiation of gland cells was observed. The glands bordering the CM consisted mainly of mucous cells and some parietal cells. At GA 41 weeks and age seven months, almost all glands in the CM and bordering the CM secreted neutral mucins. Acid mucins (predominantly sialylated mucins) were present in the foveolar epithelial cells and in some gland cells in the most proximal part of the CM. Chronic inflammation of the CM was seen after birth. HEE and mucin stains of the CM in a one week old neonate are 

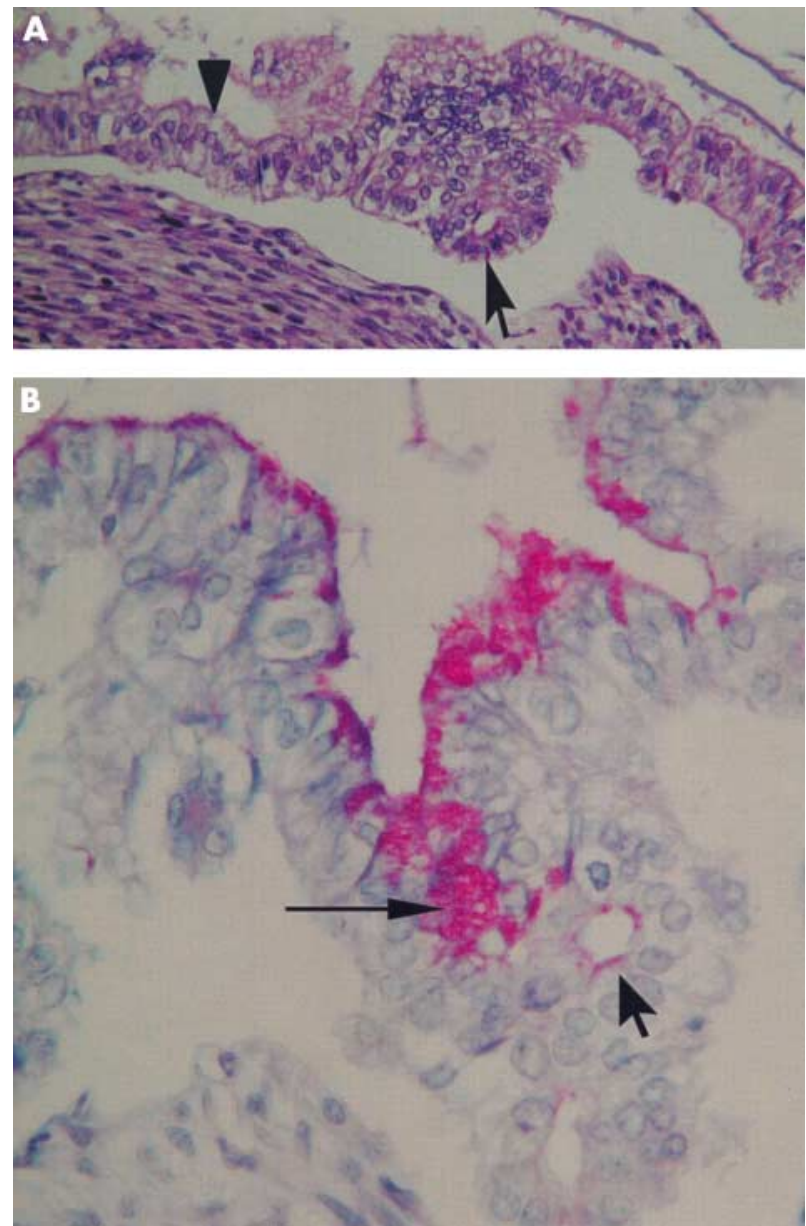

Figure 2 (A) Haematoxylin-eosin stain of the cardiac mucosa (CM) in an embryo of 13 weeks' gestational age (GA) $(\times 200)$. The arrowhead indicates the primitive stomach mucosa and the arrow a gland in the CM. (B) Mucin stain of the CM in an embryo of GA 13 weeks (alcian blue/periodic acid-Schiff 2.5; magnification $\times 400$ ). Note the different mucin content of the foveolar cells (long arrow) and the gland cells (short arrow).

shown in fig 6A and B, respectively. The location of the different mucosal types in this neonate is shown in fig 7.

\section{DISCUSSION}

Is cardiac mucosa acquired by metaplasia or is it a normal constituent of the gastro- intestinal tract? The answer to this question is important from a clinical viewpoint. If cardiac mucosa arises by metaplasia, the finding of such a mucosa in a biopsy specimen from the GOJ region may indicate the first step in a sequence from gastro-oesophageal reflux disease to adenocarcinoma. This is the view of Chandrasoma. ${ }^{10}$ In contrast, Kilgore and colleagues, ${ }^{14}$ Zhou and colleagues, ${ }^{8}$ and Glickman and colleagues ${ }^{9}$ support the concept of the gastric cardia as a normal structure which is present from birth. We performed this study to investigate the points of conflict between the above mentioned schools.

If cardiac mucosa develops during pregnancy, it is a normal structure at birth. In order to study this, we examined the GOJ in tissue samples from autopsies of embryos, fetuses, and infants. At autopsy, hiatal hernia was excluded in all cases by a careful in situ examination of the GOJ region. We studied only well preserved specimens. In each case, a maximal amount of tissue was evaluated to minimise the chance of missing short segments of cardiac mucosa. Much controversy regarding the location of the cardiac mucosa originates from the definition of the GOJ. We situated the GOJ at the level of

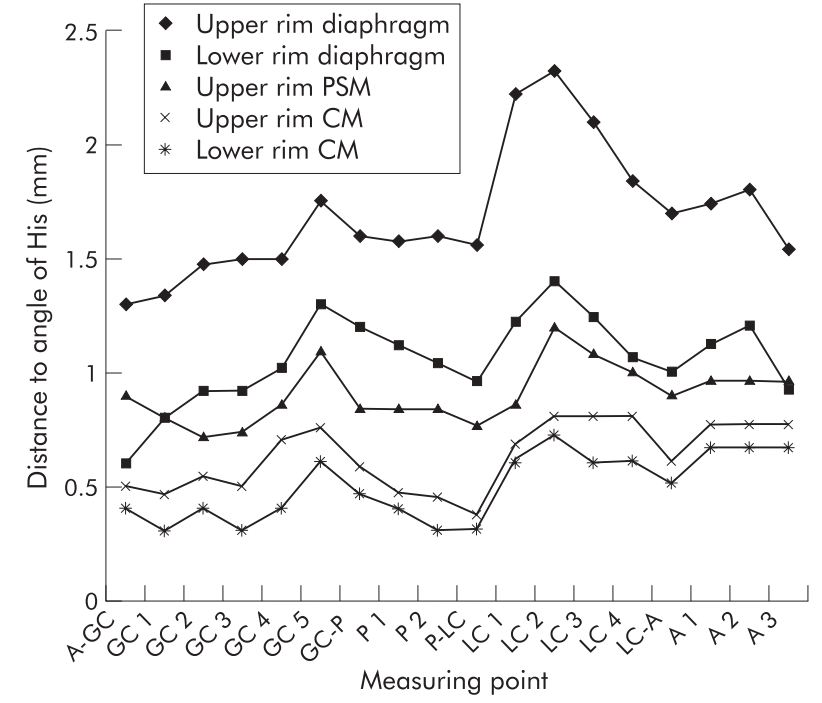

Figure 3 Location of the different mucosal types in an embryo of gestational age 13 weeks. CM, cardiac mucosa; PSM, primitive stomach mucosa (see text for explanation of measuring points).

the angle of His as this landmark could be identified easily in our material and because it has been used by anatomists, surgeons, and (implicitly) by endoscopists.

We observed cardiac mucosa in all of our specimens. Thus cardiac mucosa develops during gestation and is present at birth. In our opinion, this indicates that it is a normal structure.

Cardiac mucosa was present in our youngest subjects (two embryos of GA 13 weeks). This is in accordance with the findings of Salenius ${ }^{16}$ who found cardiac mucosa from GA 11 weeks. In his material, the cardiac glands were fully developed at a GA of 13 weeks.

Zhou and colleagues ${ }^{8}$ performed an autopsy study of 77 subjects aged between 15 weeks GA and 17 years of age. Glands lined exclusively by mucous cells were present between the oesophageal and fundic mucosa in 2/32 prenatal subjects and 22/45 postnatal subjects. These findings differ from ours. We found cardiac mucosa in all evaluated sections in all of our subjects, from GA 13 weeks to age seven months. Such a discrepancy may be explained by several factors. Firstly, it is unclear how many sections Zhou et al evaluated in each case; we evaluated a minimum of 10. Secondly, the recognition of glands may be difficult in embryos and young fetuses. We found a clear separation of pits and glands by a neck portion from GA 21 weeks. In younger subjects, the glands can be distinguished from the pits by the aspect of the nuclei of the cells lining the glands and by mucin stains. Zhou et al did not find mucous- type glands in the transitional zone in their subjects younger than 21 weeks but they did not perform mucin stains. Thirdly, according to their tabulated results, the presence of mucous or mixed glands (the latter being defined as mucous glands containing isolated parietal cells) were mutually exclusive. This probably indicates that the glands present between the oesophageal and fundic mucosa were labelled according to the most prevalent gland type (either mixed or mucous). The cardiac mucosa segment was always short in our cases. It is probable that in most cases studied by Zhou et al, the segment lined by mucous glands was shorter than the segment lined by mixed glands. Thus the denominator "mixed" would be used much more often than the denominator "mucous".

We measured the length of the cardiac mucosa and evaluated its position in all of our cases. As individual measurements at six or 18 measuring points showed a high variability, we decided to calculate means for each case and for subjects with an identical GA. 

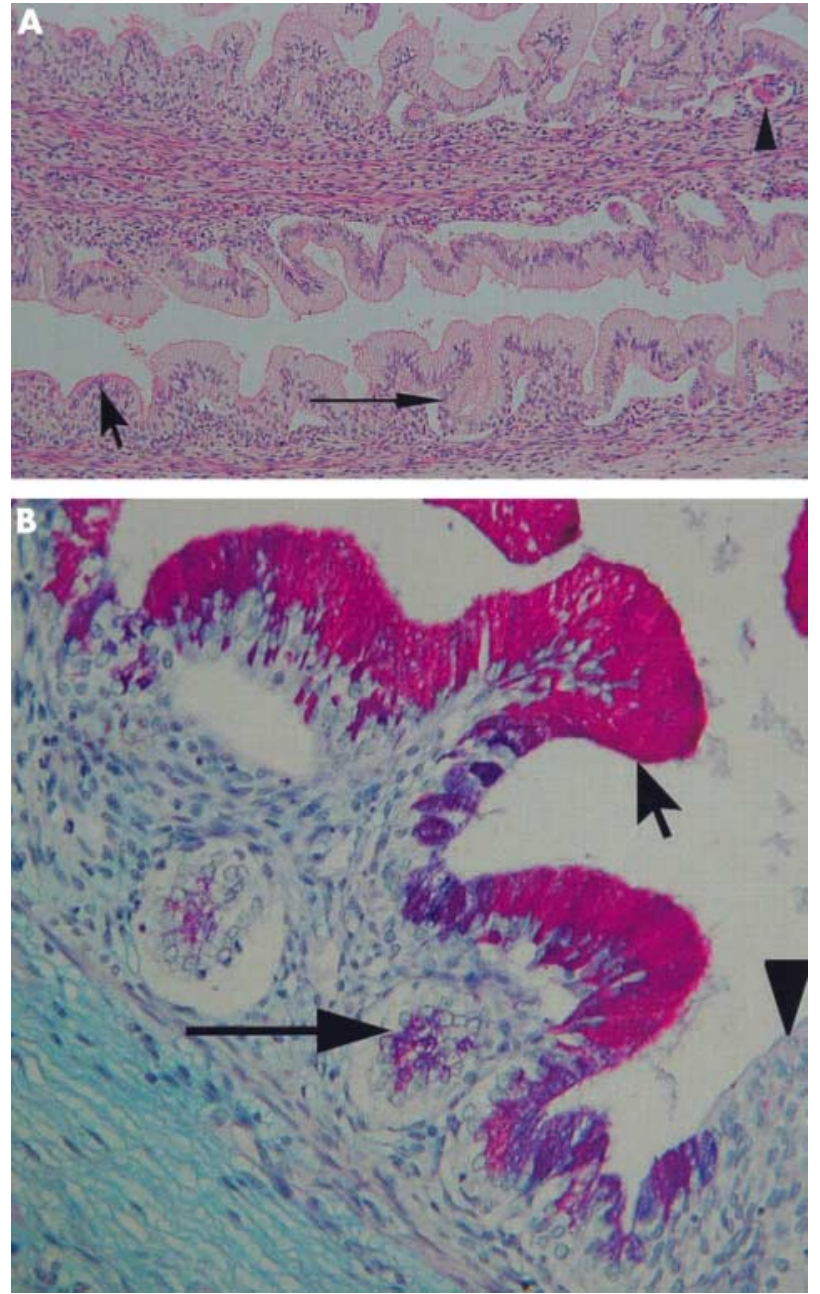

Figure 4 (A) Haematoxylin-eosin stain of the cardiac mucosa (CM) in a fetus of gestational age (GA) 24 weeks $(\times 100)$. Note the squamous oesophageal mucosa with some residual ciliated cells on top (short arrow) and the cardiac mucosa containing no parietal cells (long arrow). To the right, a cluster of parietal cells can be seen in a mixed gland (arrowhead). (B) Mucin stain of the CM in a fetus of GA 24 weeks (alcian blue/periodic acid-Schiff 2.5; magnification $\times 200$ ). The oesophageal squamous mucosa is marked by an arrowhead. The most proximal cardiac mucosal glands contain a mixture of neutral and sialylated mucins (long arrow). The surface epithelial cells contain almost exclusively neutral mucins (short arrow).

We observed that the mean length of the cardiac mucosa varied throughout gestation. It reached a maximum at 16 weeks' GA $(1.2 \mathrm{~mm})$. Its length at and after birth was very short $(0.3-0.6 \mathrm{~mm})$. This observation is in agreement with the findings of Kilgore and colleagues. ${ }^{14}$ They examined the entire GOJ in 30 paediatric autopsies (age range 16 days to 18 years). As in our study, the length of the cardiac mucosa was defined as the distance between the squamous oesophageal mucosa and the most proximal parietal cell identified. Cardiac mucosa was present in all specimens and had a mean length of $1.8 \mathrm{~mm}$ (range 1.0-4.0). Chandrasoma and colleagues ${ }^{12}$ performed a prospective autopsy study in seven paediatric patients (age range 3-18 years). The number of measurable sections across the GOJ varied between six and nine. Cardiac mucosa was absent in all sections in three cases. In the remaining cases, its length varied between 0.072 and $0.704 \mathrm{~mm}$ (mean 0.314). Our results do not offer an explanation for the absence of cardiac mucosa in some paediatric patients in this study. The observed mean length of cardiac mucosa is very close to ours however.

The second observation we made in reviewing our results relates to the position of the cardiac mucosa. When one

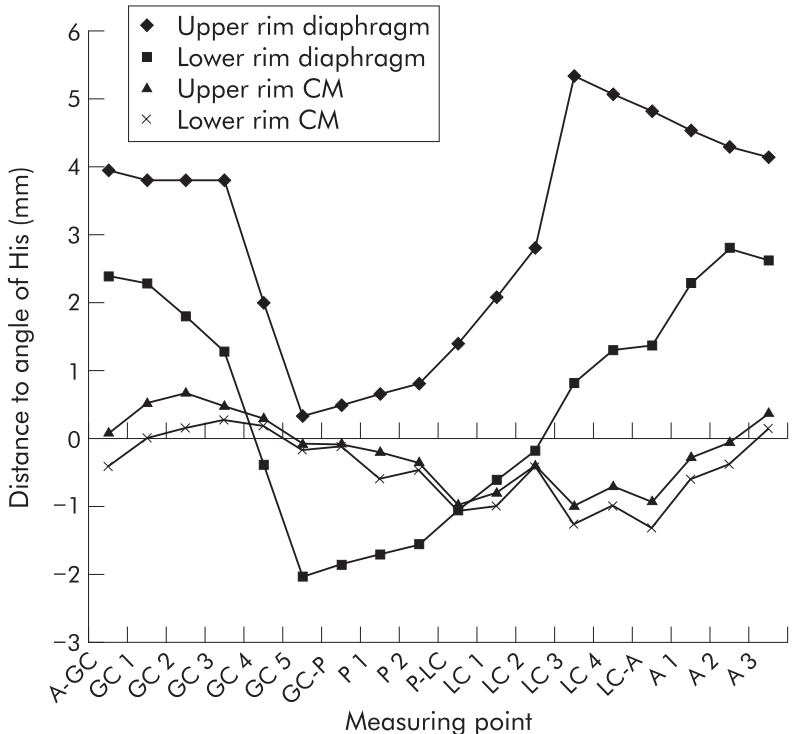

Figure 5 Location of the different mucosal types in a fetus of gestational age 24 weeks. CM, cardiac mucosa (see text for explanation of measuring points).

accepts the angle of His as a landmark for the GOJ, the cardiac mucosa was located proximal to or at the GOJ in all of our cases. Therefore, according to anatomical definitions, cardiac mucosa was located in the abdominal segment of the tubular oesophagus at birth. The distance from the cardiac mucosa to the angle of His was small however $(0.0 \mathrm{~mm}$ at 41 weeks and $0.3 \mathrm{~mm}$ at seven months). When the length of the cardiac mucosa and its distance to the angle of His are so small, one can easily draw the conclusion that the cardiac mucosa is located in the stomach. This may explain the findings by Kilgore and colleagues ${ }^{14}$ who stated that the cardiac mucosa was situated at the gastric side of the GOJ in all specimens in their paediatric patient autopsy study.

We observed an evolving mucin staining pattern in the epithelia of the cardiac mucosa. The surface and foveolar cells contained neutral and sialylated mucins at GA 13 weeks. From GA 15 weeks, acid mucins were absent in most of these cells. At GA 13 weeks, a very small amount of mucin was present in the glandular cells. They contained a moderate amount of neutral, sialylated, and sulphated mucins at GA 15 weeks. The amount of acid mucins in the glandular cells diminished from GA 15 weeks to birth. This mucin staining pattern was very similar to that of the developing pyloric mucosa. After birth, acid mucins were present in rare cells in the most proximal glands of the cardiac mucosa. Our results are in agreement with those of Ellison and colleagues ${ }^{15}$ who studied the mucin histochemistry of the developing GOJ. They found stainable mucin from GA 12 weeks. The surface epithelium contained predominantly neutral mucins at GA 12.5 weeks although $16 \%$ of cells were sialylated and $6 \%$ sulphated. Well defined glands and pits were present after 20 weeks. With their development, expression of acid mucins decreased progressively while neutral mucin increased.

In conclusion, we observed cardiac mucosa in our material from a gestational age of 13 weeks. It develops during pregnancy and is present at birth. This indicates that it is a normal structure. During gestation its mucin staining pattern is to a large degree similar to that of the developing pyloric mucosa. After birth, cardiac mucosa is present around the oesophageal perimeter, has a mean length of $0.3-0.6 \mathrm{~mm}$, and is located proximal to or at the GOJ if this is defined as the angle of His. 

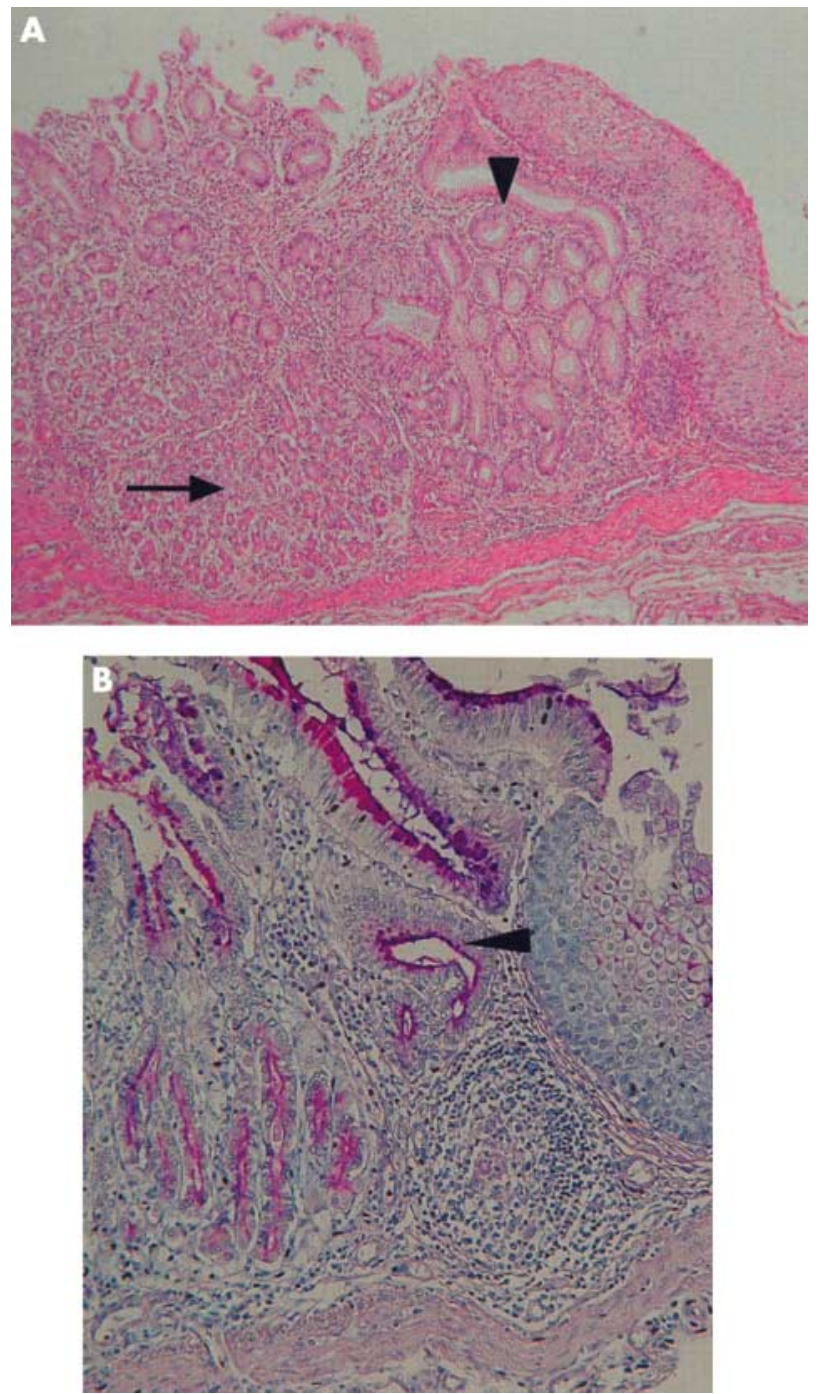

Figure 6 (A) Haematoxylin-eosin stain of the cardiac mucosa (CM) in a one week old neonate $(\times 50)$. The cardiac mucosa is in the centre (arrowhead). Mucosa containing parietal cells is present to the left (arrow). Note the presence of inflammation in the cardiac mucosa. (B) Mucin stain of the CM in a one week old neonate (alcian blue/periodic acid-Schiff 2.5 ; magnification $\times 100$ ). Note that the most proximal cardiac mucosal glands still contain acid mucins (arrowhead).

\section{Authors' affiliations}

G De Hertogh, P Van Eyken, N Ectors, K Geboes, Department of Pathology, University Hospitals, Catholic University Leuven, Leuven, Belgium

J Tack, Department of Gastro-Enterology, University Hospitals, Catholic University Leuven, Leuven, Belgium

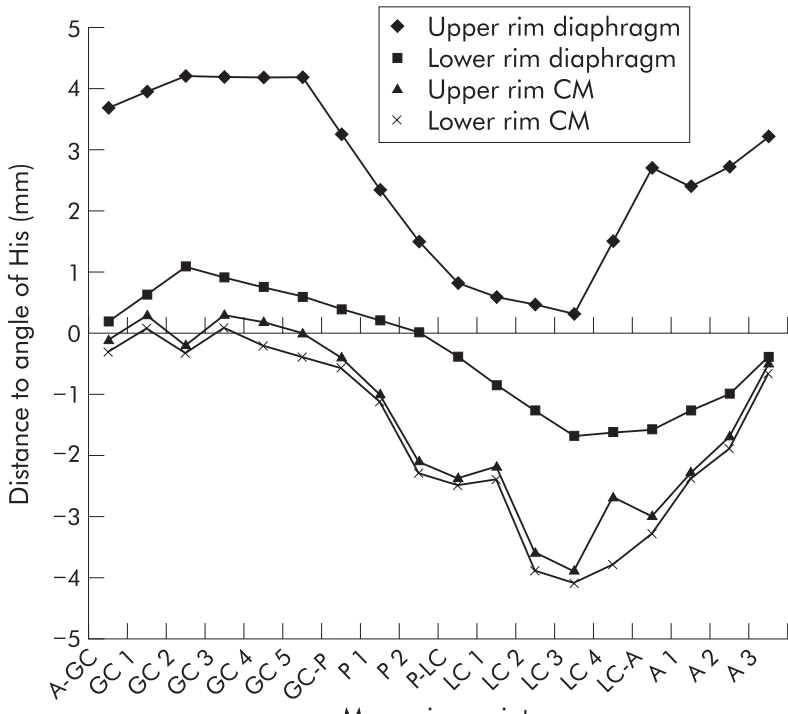

Measuring point

Figure 7 Location of the different mucosal types in a one week old neonate. CM, cardiac mucosa (see text for explanation of measuring points).

\section{REFERENCES}

1 Blot WJ, Devesa SS, Kneller RW, et al. Rising incidence of adenocarcinoma of the esophagus and gastric cardia. JAMA 1991;265:1287-9.

2 Devesa SS, Fraumeni JF. The rising incidence of gastric cardia cancer. J Natl Cancer Inst 1999;91:747-8.

3 Warwick R, Williams PL, Gray H. The stomach. In: Warwick R, Williams PL, eds. Gray's Anatomy, 36th edn. Edinburgh: Churchill Livingstone, 1980:1270.

4 Boyce HW. Endoscopic definitions of esophagogastric junction regional anatomy. Gastrointest Endosc 2000:51:586-92.

5 DeNardi FG, Riddell RH. Esophagus. In: Sternberg SS ed. Histology for Pathologists, 2nd edn. Philadelphia: Lippincott-Raven, 1997:461.

6 Owen DA. Stomach. In: Sternberg SS, ed. Histology for Pathologists, 2nd edn. Philadelphia: Lippincott-Raven, 1997:481.

7 Hayward J. The lower end of the oesophagus. Thorax 1961;16:36-41.

8 Zhou H, Alba Greco M, Daum F, et al. Origin of cardiac mucosa: ontogenic consideration. Pediatr Dev Pathol 2001;4:358-63.

9 Glickman JN, Fox V, Antonioli DA, et al. Morphology of the cardia and significance of carditis in pediatric patients. Am J Surg Pathol 2002; 26: 1032-9.

10 Chandrasoma P. Pathophysiology of Barrett's esophagus. Semin Thorac Cardiovasc Surg 1997:9:270-8.

11 Chandrasoma P, Lokuhetty DM, Demeester TR, et al. Definition of histopathologic changes in gastroesophageal reflux disease. Am J Surg Pathol 2000;24:344-51

12 Chandrasoma P, Der R, Ma Y, et al. Histology of the gastroesophageal junction-an autopsy study. Am J Surg Pathol 2000;24:402-9.

13 Chandrasoma $P$, Der R, Dalton $P$, et al. Distribution and significance of epithelial types in columnar-lined esophagus. Am J Surg Pathol 2001;25:1188-93

14 Kilgore SP, Ormsby AH, Gramlich TL, et al. The gastric cardia: fact or fiction? Am J Gastroenterol 2000;95:921-4.

15 Ellison E, Hassall E, Dimmick JE. Mucin histochemistry of the developing gastroesophageal junction. Pediatr Pathol Lab Med 1996:16:195-206.

16 Salenius P. On the ontogenesis of the human gastric epithelial cells. A histologic and histochemical study. Acta Anat (Basel) 1962;50(suppl 46): $11-70$. 